\title{
NUMERICAL MODELING OF CRACK PROPAGATION USING ZERO- THICKNESS INTERFACES ELEMENTS AND CONFIGURATIONAL MECHANICS
}

\author{
L. CRUSAT AND I. CAROL \\ Division of Geotechnical Engineering and Geo-Sciences \\ ETSECCPB (School of Civil Engineering) \\ Universitat Politècnica de Catalunya (UPC) \\ 08034 Barcelona \\ e-mail: laura.crusat@upc.edu \\ e-mail: ignacio.carol@upc.edu
}

Key words: Configurational mechanics, fracture mechanics, propagation, node-relocation

\begin{abstract}
The prediction of crack trajectories is one of the major challenges of existing numerical methods for the mechanical behavior of concrete and other quasi-brittle materials. For this purpose, this paper describes a methodology based on the use of zero-thickness interface elements equipped with a fracture-based constitutive law, combined with Configurational Mechanics theory. In the implementation developed, interface elements, initially inserted in between standard continuum elements, become realigned during the fracture propagation process on the basis of the so-called "configurational forces", which represent the gradient of the total elastic energy of the domain with respect to the initial nodal position. In this way, and unlike most methods used in crack propagation that are based on local criteria such as maximum tensile stress, in the proposed method the crack configuration that is obtained approaches a global energy minimum for the structure or domain. The performance of the method is illustrated with an example of application in which the crack path is known a priori due to structural symmetry.
\end{abstract}

\section{INTRODUCTION}

The prediction of crack initiation and propagation of quasi-brittle materials such as rocks or concrete is an essential topic in various engineering fields such as Structural or Geotechnical Engineering.

It is commonly accepted that the propagation of cracks is governed by the principles of Fracture Mechanics, which may be interpreted as establishing an energy consumption criterion per unit area of new crack created, which must be balanced with the energy relieved by elastic unloading in areas surrounding the propagating crack plus the work of external forces [1]. All these principles may be implemented numerically in the context of the FEM with cracks represented with zero-thickness interface elements [2], in which those elements are equipped with the appropriate energydriven constitutive laws [3].

In the general case, however, the above principles may be not sufficient to predict crack trajectory in cases in which that trajectory does not coincide with the mesh lines along which zero-thickness interface elements have been pre-inserted. The prediction of the most likely crack path requires the additional condition that the crack path corresponds to an energy minimum at the structural level, with respect to other alternative and energetically-balanced crack paths. Enforcing this new condition may be achieved by means of Configurational Mechanics, which is concerned with the variation of the domain elastic energy with changes in the structural geometry including crack trajectories (configuration). 
The original concepts of Configurational Mechanics were introduced in the fifties by Eshelby [4], who developed the driving forces for elastic singularities, defining the "Maxwell tensor of elasticity", also known as the energymomentum tensor or Eshelby's tensor $\boldsymbol{\Sigma}=$ WI $\mathbf{F}^{\mathrm{T}} \mathbf{P}$. This tensor represents the "driving force" on moving defects or in-homogeneities of a solid such as dislocations in plasticity. Later, the close similarities with the concept of JIntegral used in fracture mechanics motivated many researchers to study material forces application to crack propagation [5] - [6] - [7] [8]. Nevertheless, full combination of Configurational Mechanics with Fracture Mechanics in the context of the FEM is still a challenge. This paper summarizes the work developed along this line at the MECMAT group of the Geotechnical Engineering Division - UPC.

\section{BASIC CONCEPTS OF CONFIGURATIONAL MECHANICS}

Configurational Mechanics theory is based on the evaluation of energy variations caused by changes of material configuration; particularly, configurational forces are defined as the negative gradient of global elastic energy with respect to nodal location. Global energy is a function of original location as well as final node position after deformation $\mathbf{x}$, i.e. $\psi=$ $\psi(\mathbf{X}, \mathbf{x})$, this means that configurational forces are evaluated at constant $\mathbf{x}$ :

$$
\hat{\mathbf{f}}=\left.\frac{\partial \psi}{\partial \mathbf{X}}\right|_{\mathbf{x}=\mathbf{c t}}
$$

As Steinmann et al. describes in [9], configurational forces may also be interpreted as the driving forces in the kinetics of a wide range of defect types, including cracks. In the finite element context, configurational nodal forces may be obtained as the following integral over the domain:

$$
\hat{\mathbf{f}}=\int \widehat{\mathbf{B}}^{T} \boldsymbol{\Sigma} \mathrm{d} V
$$

where $\widehat{\mathbf{B}}$ is the non-symmetric version of the traditional "B" FE matrix, and $\boldsymbol{\Sigma}$ is the “configurational stress” given by Eshelby's energy-momentum tensor [3]. The following logical step is to "move" nodes in the mesh (change their coordinates) along the directions indicated by configurational forces. Similar to classical deformational analysis, node relocation mush be subject to some restrictions, such as for instance boundary restrictoins not to change the domain geometry.

Several authors [10] - [11] - [12] - [9] describe such procedures applied to continuum FE domains, generally leading to modified mesh configurations (in general preserving topology) which minimize global elastic energy. It is however not totally clear whether such modified configurations may correspond to an optimal mesh geometry. This is because, due to inevitable intrinsic FE discretization error, the minimum energy configuration does not necessarily correspond to the minimum error configuration (e.g. discretization error could be in the sense of under-evaluating elastic energy, and in that case minimum energy could actually correspond to maximum error).

In contrast to the configurational changes mentioned above, mainly altering the energy "noise" due to the intrinsic discretization error, it is also possible to identify true configurational changes modifying the domain geometry and therefore leading to "physical" energy changes. Among those, some are in general to be avoided via restriction such as the boundary changes (i.e. making the domain larger or smaller), but also those related to crack geometry, which are central to the present study on crack propagation. However, because of always existing discretization error, also physical energy variations due to "true" configurational changes will in general be affected (and may be distorted) by discretization "noise". To reduce this effect different strategies can be applied, i.e. local grid refinement (LGR) procedures to parametrize the zone of interest, or the use of higher-order elements to discretize the domain.

\section{MODEL IMPLEMENTATION}

On the basis of the above considerations and concepts, a finite element strategy has been 
developed for representing the development of cracking along non-pre-established paths [13]. It is based on the existing and broadly-tested approach of using traditional zero-thickness interface elements [14] in combination with a fracture-based constitutive law [3], while the continuum remains elastic at all times. That approach has been modified to systematically evaluate configurational forces, and re-orient newly opening interface elements on the basis of those forces (Figure 1). The change of nodal coordinates is limited to small steps depending on mesh discretization, in order to avoid excessive mesh distortion or overlap. Every time interface elements have been reoriented, a "mesh relaxation" algorithm (e.g. [15] - [16]) is applied to improve mesh quality around the modified area (Figure 2). All nodes relocated require a mapping and update of the corresponding nodal variables in order to maintain consistency of the results. This procedure is done by interpolation of the variables in the new position with respect to the previous mesh configuration (Figure 2).

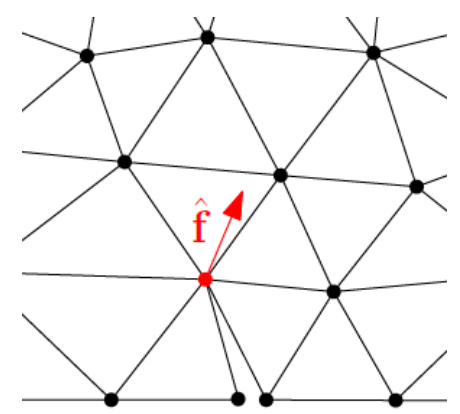

Figure 1: Representation of configurational force direction on the crack tip node

The change of location will be allowed gradually because too many simultaneous changes could destabilize the iterative process, only fracture tip nodes of the interface elements that begin to open (change of elastic to elastoplastic state) are allowed to change their configurational position. The details of this and other auxiliary procedures involved in the implementation are described in [13].

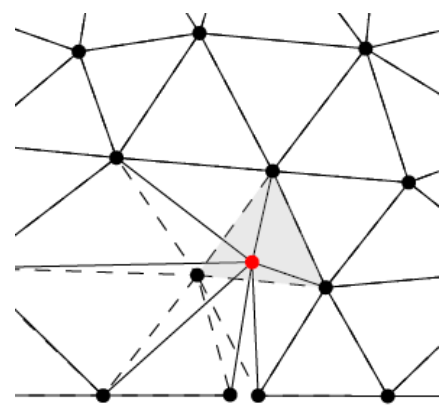

Figure 2: Mapping of the changed node, interpolation over the element on the old configuration

\section{EXAMPLES}

This section includes two examples of application. The first one consists of a square homogeneous block discretized by continuum elements and subject to uniaxial tension, and configurational changes only affect the internal continuum mesh discretization. It is a useful example to illustrate the nature of configurational forces and of the energy minimization involved in the configurational iterative process. The second example consists of a three-point bending beam in which a crack develops along the central cross-section. The mesh is initially random and will get properly aligned during configurational iterations.

\subsection{Tensile homogeneous block}

This example has been already used in configurational mechanics literature [10]. It consists of a homogeneous square elastic block with parameters $\mathrm{E}=1085.7 \mathrm{MPa}$ and $v=0.3571$. Loading consists of a tensile displacement prescribed on the top side, while displacements are fixed on the bottom side (Figure 3). The block is discretized using a regular mesh of 16 quadrilateral elements as shown in Figure 4, where the solid nodes indicate the nodes that are "configurationally fixed" (i.e. those that will not be allowed to move even if their configurational forces are not zero), while the hollow ones are "configurationally free”, i.e. they are allowed to change location through configurational iterations. 


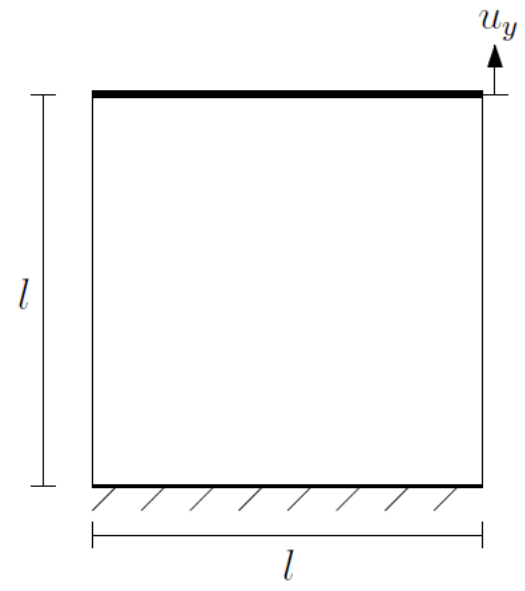

Figure 3: Deformational boundary conditions.

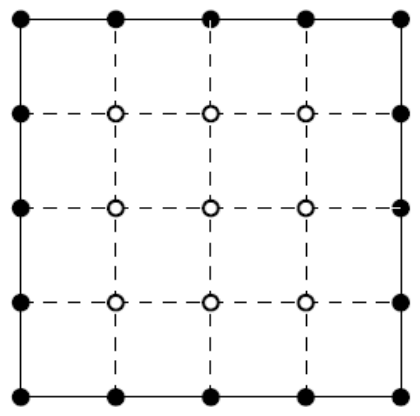

Figure 4: Finite element discretization, with indication of nodes that are "configurationally fixed" (solid) and "configurationally free” (hollow).

In this problem, configurational forces (with minus sign) are concentrated on the boundary nodes that are configurationally fixed (Figure 5 ), with such proportion that if scale is adjusted to those, the forces on inner nodes cannot be even visualized. Physically, this may be interpreted as that, if domain dimensions would be increased slightly (but final node positions would remain constant), total energy would decrease. To explain this, one has to take into account two counteracting effects. On one side, tensile deformations would be lower (because initial and final positions of nodes would be closer) and therefore volumetric energy density would be lower, but on the other side, total volume would be larger. Out of those effects, the decrease of specific energy would be dominant because is a quadratic relation. From the relocation method itself, it makes sense to fix configurational movements on the boundary so that domain dimensions will not change and only internal configurational forces (Figure 6) are allowed to change the material configuration, and therefore node relocations will reflect only the mesh rearrangement to reach an energy minimum

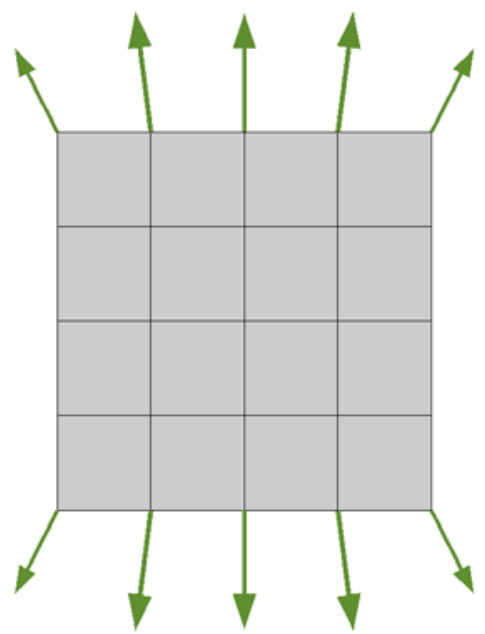

Figure 5: Configurational forces (with minus sign) on all nodes of the mesh, at the scale dictated by larger forces on the boundary nodes.

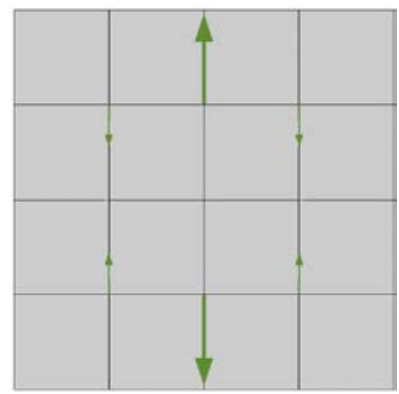

Figure 6: Same as Fig.5, but only for internal nodes, and using a larger scale forces so that forces on those nodes would be represented.

Figure 7 represents the final position of the nodes after the progressive relocation due to configurational iterations. As Figure 7 shows, nodes with larger configurational forces exhibit larger position changes. Since this optimal corresponds, not to a real geometry change of the domain but to the minimization of the FE discretization energy, which may be affected by discretization error, this solution turns out not trivial and might be different for other meshes or other element types. 


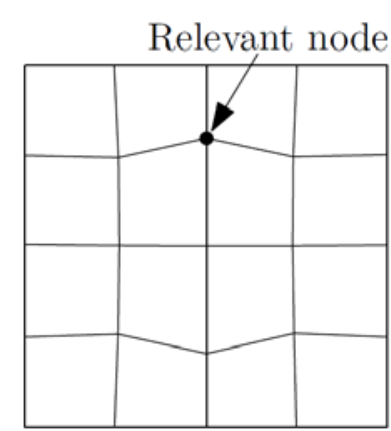

Figure 7: Mesh configuration leading to an energy minimum.

Figure 8 depicts the total energy of the mesh as a function of the $y$-coordinate position of the most relevant node in this example, and Figure 9 the norm of the configurational forces of internal nodes, also as a function of the same vertical position of the relevant node. It can be seen as the node approaches it optimal position, energy comes to a minimum and forces are reduced to zero norm.

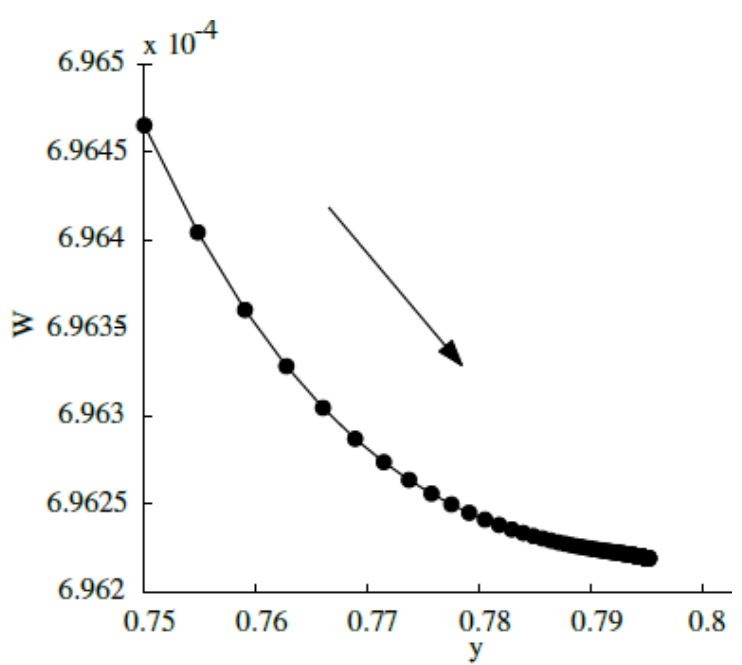

Figure 8: total block energy evolution with respect to vertical coordinate of the "relevant node".

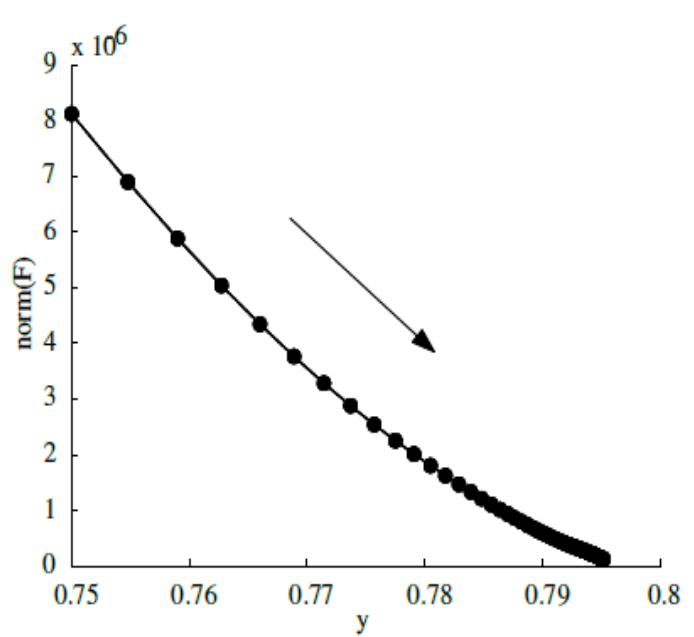

Figure 9: Norm of configurational forces with respect to the vertical coordinate of the "relevant node".

\subsection{Three-point beam bending test}

The second example of application consists of the $5 \times 1 \mathrm{~m}$ three-point bending beam test represented in Figure 10, which is discretized initially with a random triangular mesh. The three-point bending test is a good example to verify cracking along non-pre-established path because, due to symmetry, it is known that the crack path should develop vertically along the plane of symmetry, and starting from the bottom face of the beam. If the process works correctly, the initial zig-zagging mesh lines should get realigned to this vertical crack path.

Deformational boundary conditions consist of vertical constraints at the two lower corners of the beam, and a node at the middle of the upper face with horizontal displacements restricted. The loading consists of vertical displacements at this point prescribed with an increasing descending value (Figure 10).

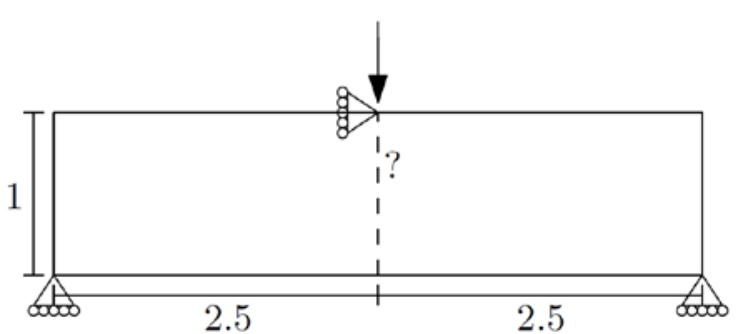

Figure 10: Boundary conditions of three-point bending test example.

The beam is discretized into triangular elements of quadratic order in order to reduce 
the discretization error (Figure 11). Due to the expected crack trajectory, and to deal with increasing complexity, a zero-thickness interface elements are pre-inserted along a single line zig-zagging around the symmetry line of the beam (red line in Figure 11).

The continuum material is assumed linear elastic (small strain), with Young's modulus value $\mathrm{E}=15000 \mathrm{MPa}$ and Poisson's ratio $v=$ 0.0.The constitutive model for the interface is the fracture-based constitutive model described in [14], with the following parameter values: normal and tangential elastic stiffness $K_{N}=K_{T}=10^{7} \mathrm{MPa} / \mathrm{m}$, friction angle $\tan \varphi=0.7$, tensile strength $\chi=3 \mathrm{MPa}$, cohesion $c=6 \mathrm{MPa}$, fracture energy mode I, $G_{f}^{I_{f}}=10^{-2} \mathrm{MPa} \cdot \mathrm{m}$, energy mode IIa, $G^{I I a} f=10^{-1} \mathrm{MPa} \cdot \mathrm{m}$ and normal stress at which dilatancy vanishes, $\sigma_{\text {dil }}=$ 30MPa.

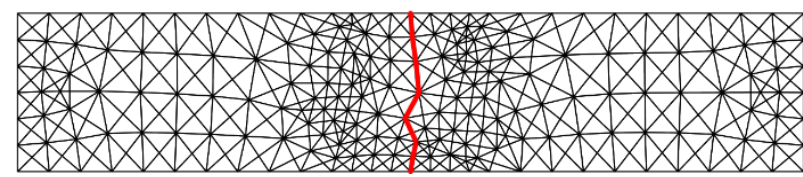

Figure 11: Initial configuration of beam bending realignment.

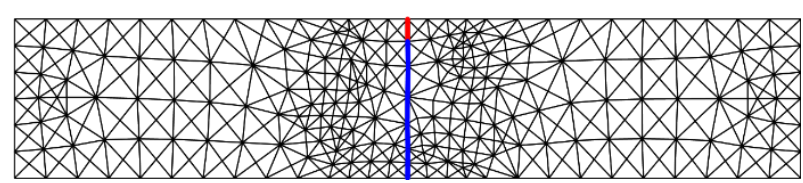

Figure 12: Final configuration of beam bending realignment.

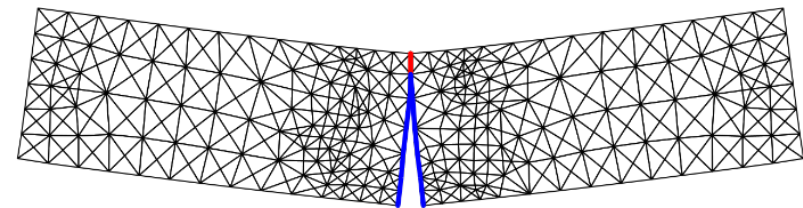

Figure 13: Three-point bending test deformation after solving the deformational and configurational problem (displacement magnification x100).

As Figure 13 depicts the failure is initialized at the center of the lower face of the beam. As expected, due to the symmetric setup the crack path propagates upwards. In Figure 12 and Figure 13 the final state of the beam is depicted. The figures show that the iterative configurational process succeeds in orienting the crack along the correct vertical direction (Figure 12).
In the figures, red lines correspond to the zero-thickness interface elements which have not started cracking yet, and therefore, in the scheme implemented they have not triggered the process of moving nodes (although some of them may have changed orientation if they share nodes with an interface which has started cracking, such is the case of the top interface in Figure 12 and Figure 13). Blue lines correspond to the interface elements that have started cracking, and therefore configurational forces may have moved them to an optimal position.

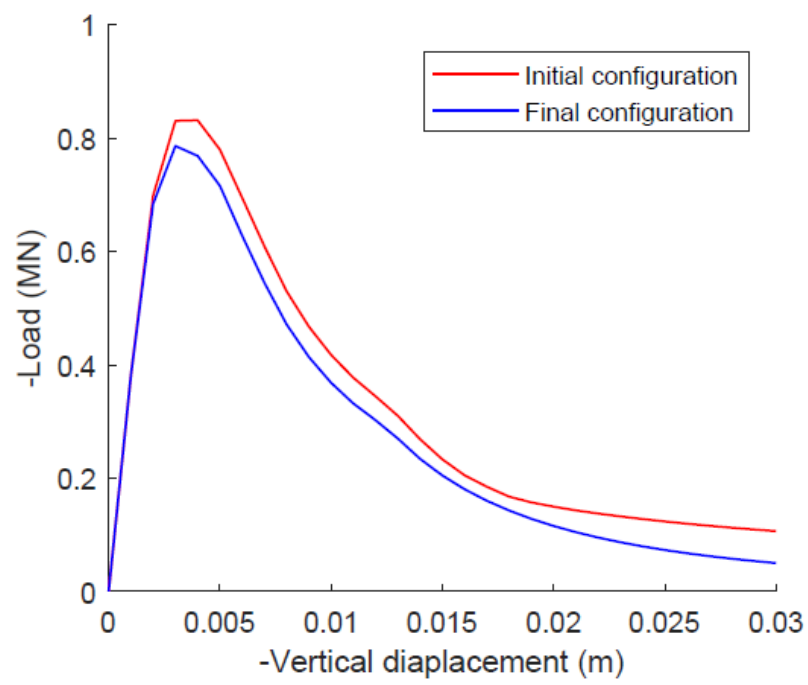

Figure 14: Three-point bending test load-displacement curves.

Figure 14 displays two load-displacement curves, the one obtained for the initial configuration fixed (mesh with the distorted crack trajectory), and the other one obtained after crack realignment (solving the FEM problem with the final configuration, mesh with the vertical crack path). As it could be expected, the load-displacement curve obtained from the final configuration exhibits a lower, more realistic peak and post-peak response. This is because in the final configuration interface elements are better oriented and therefore the crack initiates and propagates with lower applied load values.

\section{CONCLUDING REMARKS}

This article is focused on the prediction of cracking along non-preestablished path, in particular, via realignment of zero-thickness interface elements which have been pre- 
inserted in the FE mesh. The criterion used to determine crack orientation is the global energy gradient with respect to mesh nodal coordinates, information that is provided by the so-called configurational forces. Once these nodal pseudo-forces are evaluated, they indicate in which direction each node would have to be moved in order to approach a global energy minimum. The moving of the nodes is implemented in an iterative manner. In order to get a stable procedure, a careful selection is required of the nodes to be moved at a time, in this case only crack tip nodes will be moved. Moreover, small steps are used to get a gradual relocation. If the crack is propagating, physical energy changes are expected to be greater than discretization error noise; however, this condition must be ensured by proper mesh refinement or appropriate polynomial degree of the interpolation functions. Additionally, a number of complementary algorithms have been introduced to improve the strategy such as: (a) a "mesh relaxation" algorithm to avoid mesh distortion, (b) the mapping of nodal variables on their new location, and (c) a new indirect displacement control iterative procedure based on prescribed values of fracture energy dissipation. The proposed model has been preliminary verified with a three-point beam example in which a single line of zig-zagging zero-thickness interface elements succeeds in getting realigned to a final vertical crack as required by symmetry. Ongoing work aims at developing further and improving the approach described and extending its application to more complex cases of cracking and fracture.

\section{ACKNOWLEGEMENTS:}

The work was partially supported by research grants BIA2016-76543-R from MEC(Madrid), which includes FEDER funds, and 2017SGR1153 from Generalitat de Catalunya. The FPI fellowship from MEC (Madrid) to the first author is also greatfully acknowledged.

\section{REFERENCES}

[1] Bazant, Z. P., 1985. Frcture in concrete and reinforced concrete.

[2] Carol, I., López, C. M. and Roa, O., 2001. Micromechanical analysis of quasi-brittle materials using fracture-based interface elements. Int. J. Numerical Methods in Engineering. 52(1-2):193-215.

[3] Carol, I. , Prat, P. C. and Lopez, C. M.. 1997. Normal/shear cracking model: application to discrete crack analysis. Journal of engineering mechanics. 123(8): 765-773.

[4] Eshelby, J. D., 1951. The force on an elastic singularityThe force on an elastic singularity. Philosophical Transactions of the Royal Society of London A: Mathematical, Physical and Engineering Sciences. 244(877):87-112.

[5] Stumpf, H. and Le, K. C., 1990. Variational principles of nonlinear fracture mechanics,» Acta Mechanica. 83:25-37.

[6] Maugin, G. A. and Trimarco, C., 1992. Psuedomentum and material forces in nonlinear elasticity: variational formulations and application to brittle fracture. Acta Mechanica. 94:1-28.

[7] Steinmann, P., 2000. Application of material forces to hyperelastostatic fracture mechanics. I. Continuum mechanical setting. International Journal of Solids and Structures. 37(48-50):73717391.

[8] Kienzler, R. and Herrmann, G., 2012. Mechanics in material space: with applications to defect and fracture 
mechanics. Springer Science and Business Media.

[9] Steinmann, P., Scherer, M. and Denzer, R., 2009. Secret and joy of configurational mechanics: From foundations in continuum mechanics to applications in computational mechanics. ZAMM-Journal of Applied Mathematics and Mechanics. 89:614-630.

[10] Mueller, R. and Maugin, G. A., 2002. On material forces and finite element discretization. Computational mechanics. 29(1):52-60.

[11] Mueller, R., Kolling, S. and Gross, D., 2002. On configurational forces in the context of the finite element method. International Journal for Numerical Methods in Engineering. 53(7):15571574.

[12] Miehe, C. and Gürses, E., 2007. A robust algorithm for configurational-forcedriven brittle crack propagation with $\mathrm{R}$ adaptive mesh alignment. International Journal for Numerical Methods in Engineering. 72(2):127-155.

[13] Crusat, L., 2018. Numerical modeling of cracking along. PhD thesis, Universitat Politècnica de Catalunya (UPC), Barcelona.

[14] Gens, A., Carol, I. and Alonso, E. E., 1989. An interface element formulation for the analysis of soil-reinforcement interaction. Computers and Geotechnics, 7(1-2):133-151.

[15] Frey, W. H. and Field, D. A., 1991. Mesh relaxation: a new technique for improving triangulations. International Journal for Numerical Methods in Engineering, 31(6):1121-1133.
[16] Schweingruber, M. and Rank, E., 1992. Adaptive mesh generation for triangular or quadrilateral elements. In Proceedings of the First European Conference on Numerical Methods, Brussels, Belgium. 